\title{
Diagnóstico del potencial de las redes sociales como recurso didáctico en el proceso de enseñanza en época de aislamiento social
}

\section{Diagnosis of the potential of social networks as a didactic resource in the teaching process in times of social isolation}

\author{
PRADA-NÚÑEZ, Raúl ${ }^{1}$ \\ HERNÁNDEZ-SUÁREZ, César A. ${ }^{2}$ \\ MALDONADO-ESTEVEZ, Erika A. ${ }^{3}$
}

\begin{abstract}
Resumen
La investigación trató de diagnosticar la incidencia de las redes sociales como experiencias didácticas y recurso educativo. Mediante una metodología cuantitativa, se abordó un estudio descriptivo de la información a partir de 185 encuestas a estudiantes universitarios. Los resultados indican que los estudiantes confirman la utilidad de las redes sociales como recursos educativos, especialmente en el ámbito de la comunicación con los docentes. El diagnóstico muestra un abanico amplio de posibilidades de las redes sociales en el aspecto educativo: interacción entre profesor y estudiantes, compartir recursos, aprendizaje colaborativo y desarrollo de competencias comunicativas.
\end{abstract}

Palabras clave: redes sociales, enseñanza, aislamiento, COVID-19

\begin{abstract}
The research tried to diagnose the incidence of social networks as didactic experiences and educational resources in the period of isolation caused by Covid-19. By means of a quantitative methodology, a descriptive study of the information from 185 surveys to university students was approached. The results indicate that students confirm the usefulness of social networks as educational resources, especially in the field of communication with teachers. The diagnosis shows a wide range of possibilities of social networks in the educational aspect: interaction between teacher and students, sharing resources, collaborative learning and development of communication skills.

key words: social networking, education, isolation, COVID-19
\end{abstract}

\section{Introducción}

Según la UNESCO (2020a; 2020b) durante la pandemia, se comprobó el mayor uso y consumo de tecnología, ofreciendo tanto oportunidades como riesgos, a la vez que la posibilidad de ejercer una ciudadanía digital

\footnotetext{
${ }^{1}$ Docente investigador. Facultad de Educación, Artes y Humanidades. Universidad Francisco de Paula Santander. raulprada@ufps.edu.co

${ }^{2}$ Docente investigador. Departamento de Pedagogia, Andragogía, Comunicación y Multimedios. Universidad Francisco de Paula Santander. cesaraugusto@ufps.edu.co

${ }^{3}$ Docente investgador. Facultad de Educación, Artes y Humanidades. Universidad Francisco de Paula Santander. erikaalejandrame@ufps.edu.co
} 
responsable. Al respecto, el uso de la tecnología promueve una cultura participativa que fortalece el compromiso social, a través de diferentes plataformas y herramientas, como las redes sociales (Corrales, 2015).

El uso de las TIC ha desempeñado un papel fundamental en la lucha contra el coronavirus, como compartir información para salvar vidas, desacreditar la información errónea, reforzar la aplicación del derecho fundamental a la información. Igualmente, contribuye a aprovechar el uso de los medios de comunicación de interés público, los recursos educativos abiertos para el aprendizaje remoto y virtual, e interconectar a las personas (Merchant \& Lurie, 2020).

A nivel educativo, han surgido iniciativas digitales para darle continuidad a la enseñanza y aprendizaje: comunicación digital, encuentros virtuales de formación, uso de plataformas y redes sociales, han sido algunas de las estrategias utilizadas (Álvarez et al., 2020).

En los últimos años, se han producido cambios significativos en el mundo con respecto a la expansión de las redes sociales y el número de personas que las utilizan (We Are Social \& Hootsuite, 2020). Las redes sociales incluyen sitios web y aplicaciones que permiten, en una zona y un conjunto de personas que se conectan conocidas como Red Personal de Aprendizaje (Gómez-Valderrama, Hernández-Suárez y Prada-Nuñez, 2020), conocer, crear, compartir contenidos, ideas, opiniones, creencias, sentimientos y experiencias personales, sociales y educativas (Azizi, Soroush, \& Khatony, 2019). Facebook, YouTube, WhatsApp, Instagram, Twitter se encuentran entre las redes sociales virtuales más populares y comúnmente utilizadas (We Are Social \& Hootsuite, 2020). Actualmente (2020), el número de usuarios de redes sociales de forma regular en todo el mundo es superior a los 3.000 millones de personas (We Are Social \& Hootsuite, 2020). Colombia es uno de los países en desarrollo donde las redes sociales han crecido significativamente (Universidad EAFIT, 2019).

Diversas investigaciones han resaltado las dificultades comunicativas que frecuentemente se observan en el proceso educativo y que influyen de forma negativa en el mismo, por ejemplo en la investigación de Sainz (1998), se resaltan tres dificultades en la comunicación pedagógicael estilo de dirección verticalizado, la relación autoritaria que prevalece en la relación docente-estudiante y la no facilitación de la interacción comunicativa grupal en el proceso docente; por lo que el uso educativo de las redes sociales permitirá eliminar este tipo de barreras y aumentar el sentido de comunidad educativa, permitiendo a los docentes ampliar el conocimiento que tienen de sus estudiantes.

Las redes sociales desempeñan un papel crucial en los entornos de aprendizaje como un canal de comunicación clave y una fuente de apoyo social (Islas, 2011). Es importante conocer los usos, de forma que los estudiantes puedan beneficiarse de las redes sociales para su estudio y desarrollo personal.

Las redes sociales proporcionan un amplio acceso a la información y a los recursos de información, reducen las barreras a la interacción de grupo y a la comunicación (Valenzuela, 2013; Muñoz, Fragueiro y Ayuso, 2016), apoyan las actividades de aprendizaje en colaboración, alientan a los estudiantes hacia el autoaprendizaje, aumentan el compromiso y la motivación de los estudiantes, mejoran las interrelaciones de estudiantes entre sí y con sus profesores y apoyan el aprendizaje activo y social, y el desarrollo de habilidades y actitudes tales como la socialización, el trabajo en equipo o la importancia de compartir (Astudillo y Chévez, 2013; Borras, 2014; Castro, 2015; Chávez y Gutiérrez, 2015; Higueras-Rodríguez, Medina-García \& Pegalajar-Palomino, 2020).

Algunas redes sociales, como Edmodo, están específicamente diseñadas para el aprendizaje (Díaz, 2017), pero para que cualquier red social sea eficaz en el proceso educativo, debe estar bajo control y responsabilidad tanto de los docentes como de los estudiantes (Osorio, 2011; De la Hoz, Acevedo y Torres, 2015). 
Los estudiantes universitarios son unos de los usuarios más importantes las redes sociales. El uso adecuado de las mismas tiene consecuencias académicas, sociales y de salud positivas para los estudiantes. Los bajos logros académicos pueden ser una de las consecuencias por el uso inadecuado de las redes sociales para los estudiantes.

Así pues, teniendo en cuenta el papel fundamental de las redes sociales en el desempeño académico de los estudiantes, y también para ampliar el conocimiento sobre los efectos de las redes sociales en estudiantes universitarios, se diseñó y llevó a cabo el presente estudio, cuyo propósito fue investigar el alcance de uso de las redes sociales entre los estudiantes universitarios.

\section{Metodología}

\subsection{Enfoque y diseño de la investigación}

Esta investigación se orienta desde el enfoque cuantitativo - descriptivo, como lo afirma Kothari (2004) puesto que incorpora procedimientos y métodos tendientes a maximizar la objetividad de los datos por medio del uso de tablas de frecuencia, descriptivo estadísticos y algunas representaciones gráficas. El diseño de la investigación se ajusta a las investigaciones de campo puesto que la recolección de los datos se realizará directamente de la fuente primaria sin intervenir en sus respuestas (Arias, 2012).

\subsection{Población y muestra}

La población objeto de estudio es la totalidad de estudiantes matriculados durante el primer semestre del año 2020 de una Institución de Educación Superior privada de la ciudad de San José de Cúcuta (Norte de Santander) en la que se ofrecen siete programas de pregrado pertenecientes a tres facultades (Ingeniería, Salud y Humanidades). Para la recolección de los datos se aplicó un muestreo probabilístico bajo la técnica de muestreo sistemático, obteniendo un tamaño de muestra de 185 estudiantes a partir de una población finita de 3264 estudiantes, definiendo un error del $6 \%$ y un nivel de confianza del $95 \%$. Se recurre al muestreo sistemático puesto que de cada programa académico se genera una lista de estudiantes y se organizan uno debajo del otro, de tal forma que permite seleccionar estudiantes en todos los semestres académicos de cada programa, ganando diversidad de puntos de vista.

\subsection{Instrumento}

Con el fin de evaluar en los estudiantes la percepción sobre la factibilidad del uso de las redes sociales como recurso didáctico en el desarrollo de los cursos académicos, se recurrió a la aplicación de un cuestionario, el cual contenía 24 ítems distribuidos en tres bloques: perfil general del informante; conocimiento, usos y riesgos de las redes sociales; y percepción sobre el uso didáctico de las redes sociales en el proceso de enseñanza. El instrumento se aplicó en la semana de exámenes previa finalización del primer semestre académico del 2020 en el que, debido a la declaratoria de aislamiento social obligatorio, se dio la necesidad de migrar de clases presenciales a asistidas por tecnología. Dado lo repentino de la necesidad, este primer semestre se asumió como un espacio de aprendizaje y esta investigación surge como un estudio exploratorio con el fin de implementar planes de mejoramiento para el segundo semestre académico del 2020. El instrumento contó con la validación mediante juicio de expertos.

\subsection{Procesamiento y análisis de los datos}

Una vez que se diseñó el cuestionario en su versión on line, se compartió a los correos institucionales de los estudiantes integrantes de la muestra y se les invitó a compartir su experiencia y diligenciar el cuestionario. Completada la muestra, los datos fueron descargados en formato Excel, para posteriormente ser exportados al software SPSS v25 en donde se creó la data, y luego se realizó el análisis descriptivo de cada sección del cuestionario reportando resultados en porcentajes acompañados de sus respectivas interpretaciones. 


\section{Resultados}

\subsection{Perfil general}

En cuanto al perfil del informante, se determinó que existía predominio del género femenino concentrando el $63 \%$ de los casos, con edades entre 20 y 39 en el $60 \%$ de los casos, mientras que el porcentaje restante se concentra entre 15 y 19 años. Los programas de la Facultad de Ciencias Humanas concentran el 52\% de los encuestados, seguido por la Facultad de Ingeniería con el 35\% de los casos y el porcentaje restante corresponde a los programas de la Facultad de Salud. En cuanto al uso de redes sociales, el $98 \%$ afirmó que las usaban diariamente, dedicando a WhatsApp entre cinco y diez horas al día, mientras que a aplicaciones como Facebook, Youtube, Instagram, Twitter y Snapchat, le dedica menos de cinco horas al día.

\subsection{Conocimientos, usos y riesgos de las redes sociales}

Al indagar entre los informantes sobre las redes sociales que más utilizan se destaca la aplicación de WhatsApp como la más usada con una cobertura del $45 \%$ de los informantes, seguida de Facebook y Youtube con porcentajes del $23 \%$ y $21 \%$ respectivamente. Se resalta la aplicación de Tik Tok como una red emergente que cada día va ganando adeptos.

Una vez identificadas las redes sociales con mayor aceptación entre los informantes, se indagó sobre los usos que realizaban en ellas. La información ha sido consignada en la Tabla 1.

Tabla 1

Usos que le dan a las principales redes sociales

\begin{tabular}{|c|c|}
\hline Red Social & Usos \\
\hline Facebook & $\begin{array}{l}\text { El } 47 \% \text { la usa como diversión mediante el acceso a juegos y aplicaciones, el } 36 \% \text { la utiliza } \\
\text { como medio de comunicación bien sea entre amigos o para buscar nuevas amistades } \\
\text { mientras chatea, lee estados o revisa publicaciones, y el } 17 \% \text { restante recurre a ella para la } \\
\text { búsqueda de información que apoye su proceso de aprendizaje. }\end{array}$ \\
\hline WhatsApp & $\begin{array}{l}\text { El 71\% la utiliza como medio de comunicación (chat, vídeo o audio) con personas conocidas } \\
\text { a través del cual comparten sentimientos, vivencias o como medio para iniciar nuevas } \\
\text { amistades. El porcentaje restante afirma que lo usa como medio de entretenimiento o } \\
\text { diversión, a través de la revisión de estados, algunos se comentan y otros no. }\end{array}$ \\
\hline Youtube & $\begin{array}{l}\text { El 75\% de los informantes asegura que utiliza este recurso como principal medio para la } \\
\text { búsqueda de información que puede ser de carácter general o con fines académicos para } \\
\text { luego ser compartido a sus amigos, mientras que el porcentaje restante asegura que lo } \\
\text { utiliza como medio de diversión principalmente para ver videos musicales o publicar los } \\
\text { suyos. }\end{array}$ \\
\hline Instagram & $\begin{array}{l}\text { En opinión del } 90 \% \text { de los informantes, esta red la usa principalmente como medio de } \\
\text { comunicación en el cual interactúa con personas conocidas o para leer publicaciones o } \\
\text { estados de personas de interés nacional e internacional. El } 10 \% \text { restante, lo usa } \\
\text { principalmente como medio de diversión en actividades de ocio tales como compartir } \\
\text { contenido o fotografías. }\end{array}$ \\
\hline Twitter & $\begin{array}{l}\text { El } 12 \% \text { afirman que utilizan esta red social para buscar información de actualidad, mientras } \\
\text { que el } 88 \% \text { restante lo usa como medio de comunicación a partir de la revisión de } \\
\text { publicaciones o estados, expresa sus ideas de rechazo o apoyo a ciertos temas de } \\
\text { actualidad. }\end{array}$ \\
\hline Snapchat & $\begin{array}{l}\text { La totalidad de personas que lo usa, manifiesta que lo hace sólo por diversión en } \\
\text { actividades de ocio y tiempo libre, tales como la lectura de publicaciones y estados o para } \\
\text { publicar fotos. }\end{array}$ \\
\hline
\end{tabular}

Fuente: Elaboración propia 
Como se evidencia en la Tabla 1, dentro del grupo de estudiantes encuestados se observan ciertas especialidades en las redes sociales que ellos utilizan, como por ejemplo, WhatsApp es el medio de comunicación en tiempo real preferido puesto que ofrece las opciones de voz, video y mensajería instantánea; así mismo Youtube es la red preferida para compartir videos o para consultar material audiovisual de toda índole, y las demás redes citadas son usadas como medio de comunicación para mensajería asincrónica, publicación de fotos o diversas actividades de ocio.

\subsection{Percepción sobre el uso didáctico de las redes sociales en el proceso de enseñanza}

En la tercera sección del instrumento se pretendía explorar las percepciones que los estudiantes tenían sobre el posible uso didáctico de las diversas redes sociales en apoyo al proceso educativo. Por tal motivo al preguntarle al grupo de estudiantes sobre si creía que las redes sociales podrían tener algún uso educativo, se pudo determinar que el $99 \%$ de ellos respondió que sí, pero de ese porcentaje, el 54\% afirma que durante el primer semestre del año 2020 recurrió a algunas de ellas como recurso de apoyo a la labor docente, siendo el WhatsApp la más usada como medio de comunicación entre el docente y los estudiantes de cada curso.

En la Tabla 2 se resumen los usos sugeridos por los estudiantes encuestados en función de las redes sociales con mayor aceptación entre ellos.

Tabla 2

Usos educativos sugeridos para las principales redes sociales

\begin{tabular}{|l|l|}
\hline \multicolumn{1}{|c|}{ Red Social } & \multicolumn{1}{c|}{ Usos } \\
\hline Facebook & $\begin{array}{l}\text { Consultar información sobre los diversos contenidos, debatir sobre algún tema de interés, } \\
\text { compartir información académica, intercambiar apuntes o resolver dudas, realizar } \\
\text { trabajos de investigación en los cuales se requiera el diligenciamiento de encuestas. }\end{array}$ \\
\hline WhatsApp & $\begin{array}{l}\text { Intercambiar apuntes y contenido de clase, realizar tutorías con el profesor o con los } \\
\text { diferentes compañeros de clase con el fin de resolver dudas o ampliar orientaciones, } \\
\text { debatir sobre un tema en particular a partir de los grupos que podrían funcionar como } \\
\text { foros. }\end{array}$ \\
\hline Youtube & $\begin{array}{l}\text { Consultar contenidos académicos en formato audiovisual, resolver dudas, realizar } \\
\text { exposiciones sobre temas de clase. }\end{array}$ \\
\hline Instagram & Resolver dudas, consultar contenido e intercambiar apuntes de clase. \\
\hline Twitter & $\begin{array}{l}\text { Consultar contenido, debatir sobre un tema de interés, compartir información académica, } \\
\text { aclarar dificultades en el desarrollo de un tema. }\end{array}$ \\
\hline
\end{tabular}

Fuente: Elaboración propia.

A partir de esas posibles opciones de aplicación de las diversas redes sociales en las diferentes actividades académicas que se pueden sugerir en el desarrollo de un curso, se les preguntó si han creado grupos con fines académicos, ante lo cual el $75 \%$ respondió que las redes sociales en esta época de aislamiento social obligatorio le ha sido de mucho apoyo gracias a los diversos grupos en los que participa, pero aclara que tiene grupos para cada asignatura en donde interactúa con el docente y aparte ha creado diversos grupos sólo de compañeros de clase.

Debido al desarrollo de actividades asistidas con tecnología en los distintos niveles educativos, se han puesto en evidencia muchas debilidades en cuanto a la robustez del servicio de internet que se ofrece, al tiempo de las debilidades en cuanto a poder garantizar la seguridad y privacidad de la información que en redes sociales se comparte. En este sentido la totalidad de los encuestados manifiesta ser consciente de los riesgos a los que se expone con el uso de las redes sociales, pero afirma que trata de evitar publicar información personal o susceptible que le pueda perjudicar a futuro, sin dejar de reconocer el impacto que ellas pueden tener en la actual situación que vivimos debido a la pandemia de la COVID-19. 
Por otra parte, el $75 \%$ afirma que no han tenido ningún tipo de inconveniente en el uso de las diversas redes sociales, mientras que el porcentaje restante afirma haber sufrido de ciberacoso o de ciberbullying.

Finalmente, en la Tabla 3 se intentó clasificar los usos que los estudiantes encuestados le han dado a las diversas redes sociales, durante esta época de aislamiento social obligatorio.

Tabla 3

Usos dados a las principales redes sociales

durante el aislamiento social obligatorio

\begin{tabular}{|l|c|c|c|}
\hline \multirow{2}{*}{ Red Social } & \multicolumn{3}{|c|}{ Usos } \\
\cline { 2 - 4 } & Estudio & Ocio & Otras \\
\hline Facebook & $30 \%$ & $35 \%$ & $35 \%$ \\
\hline WhatsApp & $66 \%$ & $20 \%$ & $14 \%$ \\
\hline Youtube & $52 \%$ & $43 \%$ & $5 \%$ \\
\hline Instagram & $3 \%$ & $65 \%$ & $32 \%$ \\
\hline Twitter & $7 \%$ & $67 \%$ & $26 \%$ \\
\hline Snapchat & $2 \%$ & $76 \%$ & $22 \%$ \\
\hline \multicolumn{3}{|c|}{ Fuente. Elaboración propia } \\
\hline
\end{tabular}

Fuente: Elaboración propia

Dentro de los otros usos que sugieren los informantes en época de aislamiento social de las redes sociales se destaca principalmente el comercio electrónico, puesto que ha surgido como una actividad opcional en la que se puede ofertar todo tipo de productos llegando a un número ilimitado de clientes potenciales sin necesidad de pagar impuestos o tener registro de Cámara de Comercio, surgiendo como una alternativa para la generación de ingresos, por lo cual les ha llevado a abrir cuentas en más de una red social, pero siendo Facebook y WhatsApp las preferidas.

\subsection{Discusión}

El aumento del uso de las redes sociales es una de las consecuencias de mayor alcance de la pandemia COVID19 (Organización Panamericana de la Salud - OPS, 2020; Merchant \& Lurie, 2020). Este estudio realizó un diagnóstico que describe el uso de redes sociales durante el periodo del confinamiento obligatorio, con una muestra compuesta por estudiantes universitarios. Los resultados apuntan a que el aspecto comunicativo de las redes sociales abre un nuevo espacio digital para los estudiantes y sus interacciones con los docentes y otros actores educativos, muy superior al de los medios tradicionales (Merchant \& Lurie, 2020).

Por otro lado, la investigación educativa relacionada con el uso de las redes sociales en el aspecto educativo ha demostrado la mejora significativa de la práctica pedagógica (Carneiro, Toscano y Díaz, 2011). Esto hace parte de la necesidad de investigar procesos pedagógicos novedosos, y una de las herramientas esenciales en la actualidad son las redes sociales, dada la relevancia que tienen en el impacto de las actividades de los estudiantes (Chai \& Fan, 2016). Además de que son un medio que permite conocer el proceso de aprendizaje de los estudiantes (Pérez, Ortiz y Flores, 2015).

Al igual que otras tecnologías digitales, las redes sociales no se desarrollaron ni se concibieron originalmente con fines de aprendizaje, pero han evolucionado y se han adaptado para convertirse un recurso indispensable para la comunidad educativa (Arévalo, Gamboa y Hernández, 2016; Hernández, Prada y Ramírez, 2018). Las repercusiones de la utilización de las redes sociales en las escuelas y las actividades académicas de hoy en día son fundamentales; por ejemplo, los profesores utilizan cada vez más las redes sociales para innovar las prácticas de enseñanza/aprendizaje (Islas, 2011). Las usan docentes, padres de familia y estudiantes de todos los niveles educativos quienes se pueden conectar en redes personales de aprendizaje, que tienen un aspecto social que depende de relaciones e interacciones a través de las TIC (Gómez-Valderrama, Hernández-Suárez y Prada-Nuñez, 
2020), convirtiéndolas en un medio socio-relacional debido a que existe mayor conectividad comunicativa (Espinel-Rubio, Hernández-Suárez y Rojas-Suárez, 2020a).

Las redes sociales pueden convertirse en un medio de comunicación para fortalecer las interacciones de los miembros de las instituciones educativas. El entretenimiento, la búsqueda de información y el aprendizaje son algunas de las principales motivaciones de los estudiantes para utilizar las redes sociales (Espinel-Rubio, Hernández-Suárez, y Rojas-Suarez, 2020b).

Lo resultados de este trabajo hablan del uso y la importancia de las redes sociales en estudiantes universitarios. Esto es coherente con otras investigaciones como las de Kircaburun, Alhabash, Tosuntaş \& Griffiths (2020), que evidencia que el uso de redes sociales entre estudiantes universitarios se convierte en recursos didácticos para la educación superior.

\section{Conclusiones}

El desarrollo acelerado que han alcanzado las TIC las ha llevado a ser parte de muchos aspectos o actividades de la vida de las personas. En algún momento la educación se había mantenido con un proceso de apropiación mesurado en algunos casos por la resistencia a romper paradigmas tradicionales de orientación y otros tantos, por las dificultades de acceso a muchos de estos recursos por parte de algunas familias. Pero con la declaratoria de aislamiento social obligatorio en el mes de marzo, como mecanismo de prevención para desacelerar el nivel de contagio de la COVID-19, la totalidad de centros educativos junto con sus actores debieron migrar sin mediación alguna a la utilización de muchos de estos recursos TIC.

Ante la apremiante necesidad de seguir garantizando el proceso de enseñanza, las instituciones de educación superior debieron continuar y finalizar con un semestre académico que inicialmente se había pactado bajo modalidad presencial. Esta situación llevó a la incorporación de diversas aplicaciones las cuales en muchos casos se conocían y de inmediato empezaban a formar parte del acto educativo, pero quedaron muchas limitaciones sin atender en los estudiantes.

Siendo la principal motivación que llevó a la realización de esta investigación, se pudo evidenciar que en los estudiantes encuestados existe un amplio uso de diversas redes sociales, las cuales podrían ser incorporadas por sus docentes como mecanismos opcionales de acompañamiento y desarrollo de sus clases. Por ello se concluye que la utilización de herramientas innovadoras es un elemento clave para mejorar el proceso educativo y, por consiguiente, el uso de las redes sociales para mejorar no sólo el aprendizaje de los estudiantes, sino también para mejorar las expectativas del profesor.

\section{Referencias bibliográficas}

Álvarez, H., Arias, E., Bergamaschi, A., López, A., Noli, A., Ortiz, M., . . Viteri, A. (2020). La educación en tiempos del coronavirus: Los sistemas educativos de América Latina y el Caribe ante COVID-19. https://publications.iadb.org/publications/spanish/document/La-educacion-en-tiempos-del-coronavirusLos-sistemas-educativos-de-America-Latina-y-el-Caribe-ante-COVID-19.pdf

Arévalo, M. A., Gamboa, A. A., y Hernández, C. A. (2016). Políticas y programas del sistema educativo colombiano como marco para la articulación de las TIC. Aletheia, 8(1), 12-31. https://aletheia.cinde.org.co/index.php/ALETHEIA/article/view/302

Arias, F. (2012). El Proyecto de Investigación. Introducción a la metodología científica. Caracas: Editorial Episteme. 
Astudillo, M. P., y Chévez, F. (2013). Las redes sociales: un apoyo para docentes y estudiantes en el proceso educativo universitario. Educación y tecnología, (4), 30-42.

http://revistas.umce.cl/index.php/edytec/article/view/179

Azizi, S. M., Soroush, A., y Khatony, A. (2019). The relationship between social networking addiction and academic performance in Iranian students of medical sciences: a cross-sectional study. BMC Psychol, 7(28). https://doi.org/10.1186/s40359-019-030

Borras, O. (7 de agosto de 2014). Uso de las Redes sociales en Educación. https://urjconline.atavist.com/usoredes-sociales-educacion-2

Carneiro, R., Toscano, J., y Díaz, T. (2011). Los desafíos de las TIC para el cambio educativo. Madrid: Organización de Estados Iberoamericanos para la Educación, la Ciencia y la Cultura (OEI) - Fundación Santillana.

Castro, O. (2015). Social Media as Learning Tool in Higher Education: The case of Mexico and South Kore. Sinéctica, (44), 1-16. https://sinectica.iteso.mx/index.php/SINECTICA/article/view/160

Chai, J., \& Fan, K. K. (2016). Mobile inverted constructivism: education of interaction technology in social media. Eurasia Journal of Mathematics, Science and Technology Education, 12(5), 1425-1442. https://www.ejmste.com/article/mobile-inverted-constructivism-education-of-interaction-technology-insocial-media-4552

Chávez, I. L., y Gutiérrez, M. (2015). Redes sociales como facilitadoras del aprendizaje de ciencias exactas en la educación superior. Apertura, 7(2), 49-61. http://www.udgvirtual.udg.mx/apertura/index.php/apertura/article/view/698

Corrales, R. (2015). Impacto de las Redes Sociales sobre la Participación Ciudadana en Procesos Electorales y la Democracia: Caso de Costa Rica. Buenos Aires: CLACSO.

De la Hoz, L. P., Acevedo, D., y Torres, J. (2015). Uso de Redes Sociales en el Proceso de Enseñanza y Aprendizaje por los Estudiantes y Profesores de la Universidad Antonio Nariño, Sede Cartagena. Formación universitaria, 8(4), 77-84. https://doi.org/10.4067/S0718-50062015000400009

Díaz, J. E. (2017). Edmodo como herramienta virtual de aprendizaje. INNOVA Research Journal, 2(10), 9-16. https://revistas.uide.edu.ec/index.php/innova/article/view/259/1306

Espinel-Rubio, G., Hernández-Suárez, C., \& Rojas-Suárez, J. (2020a). Las TIC como medio socio-relacional: un análisis descriptivo en el contexto escolar con adolescentes de educación media. Revista de Investigación, Desarrollo e Innovación, 11(1), 99-112. https://doi.org/10.19053/20278306.v11.n1.2020.11686

Espinel-Rubio, G., Hernández-Suárez, C., y Rojas-Suarez, J. (2020b). Usos, apropiaciones y nuevas prácticas comunicativas de los usuarios adolescentes de Facebook. Saber, Ciencia y Libertad, 15(1), 280-296. https://doi.org/10.18041/2382-3240/saber.2020v15n1.6316

Gómez-Valderrama, C., Hernández-Suárez, C., y Prada-Núñez, R. (2020). La zona de posibilidades en el proceso de aprendiencia del residente digital: Un análisis cualitativo en la Red de experiencias Matemáticas de Norte de Santander: zona de posibilidades en el proceso de aprendiencia del residente digital. Educación y Humanismo, 22(38). https://doi.org/10.17081/eduhum.22.38.3688

Hernández, C., Prada, R. y Ramírez, P. (2018). Perspectivas actuales de los docentes de Educación Básica y Media acerca de la aplicación de las Competencias Tecnológicas en el aula. Revista ESPACIOS, 39(43), 19. http://www.revistaespacios.com/a18v39n43/a18v39n43p19.pdf 
Higueras-Rodríguez, L., Medina-García, M., \& Pegalajar-Palomino, M. C. (2020). Use of Twitter as an Educational Resource. Analysis of Concepts of Active and Trainee Teachers. Education Sciences, 10(8), 200. https://doi.org/10.3390/educsci10080200

Islas, C. (2011). Uso de las redes sociales como estrategias de aprendizaje. ¿Transformación educativa? Apertura, 3(2). https://www.udgvirtual.udg.mx/apertura/index.php/apertura/article/view/198/213

Kircaburun, K., Alhabash, S., Tosuntaş, S. B., \& Griffiths, M. D. (2020). Uses and gratifications of problematic social media use among university students: A simultaneous examination of the Big Five of personality traits, social media platforms, and social media use motives. International Journal of Mental Health and Addiction, 18(3), 525-547. https://doi.org/10.1007/s11469-018-9940-6

Kothari, C. R. (2004). Research Methodology: Methods and Techniques. New Delhi: New Age International

Merchant, R. N., \& Lurie, N. (2020). Social Media and Emergency Preparedness in Response to Novel Coronavirus. JAMA, 323(20), 2011-2012. https://doi.org/10.1001/jama.2020.4469

Muñoz, M., Fragueiro, M. S., y Ayuso, M. J. (2016). La importancia de las redes sociales en el ámbito educativo. Escuela abierta, 16, 91-104. http://www.ceuandalucia.es/escuelaabierta/pdf/articulos_ea16_pdf/redes\%2091-104.pdf

Organización Panamericana de la Salud (OPS). (2020). Las redes sociales y COVID-19: La contribución de BIREME. https://www.paho.org/bireme/index.php?option=com_content\&view=article\&id=479:redessociais-e-covid-19-a-contribuicao-da-bireme\&ltemid=183\&lang=es

Osorio, A. (2011). Problemáticas educativas, docentes investigadores y política pública educativa de Bogotá. Bogotá: Instituto para la Investigación Educativa y el Desarrollo Pedagógico, IDEP.

Pérez, M., Ortiz, M. G., y Flores, M. M. (2015). Redes sociales en Educación y propuestas metodológicas para su estudio. Ciencia, docencia y tecnología, 26(50), 188-206.

http://www.pcient.uner.edu.ar/index.php/cdyt/article/view/53

Sainz, L. (1998). La comunicación en el proceso pedagógico: algunas reflexiones valorativas. Educación Médica Superior, 12(1), 26-34. http://scielo.sld.cu/pdf/ems/v12n1/ems04198.pdf

UNESCO. (9 de junio de 2020a). Oportunidades y riesgo de internet en tiempos de aislamiento. https://es.unesco.org/news/oportunidades-y-riesgos-internet-tiempos-aislamiento

UNESCO. (9 de junio de 2020b). Riesgos y oportunidades de internet en tiempos de aislamiento. https://es.unesco.org/sites/default/files/ed-riesgosoportunidadesinternet-covid-esp.pdf

Universidad EAFIT. (11 de abril de 2019). EAFIT y TigoUne promueven el uso responsable de las TIC en Colombia. https://www.eafit.edu.co/noticias/revistauniversidadeafit/173/eafit-tigoune-responsabilidad-tic

Valenzuela, R. (2013). Las redes sociales y su aplicación en la educación. Revista digital universitaria, 14(4). http://www.revista.unam.mx/vol.14/num4/art36/index.html

We Are Social \& Hootsuite. (2020). Digital 2020. Global Digital Overview. Essential insights into how people around the world use the internet, mobile devices, social media, and ecommerce. https://wearesocial.com/digital-2020

Esta obra está bajo una Licencia Creative Commons Attribución-NoCommercial 4.0 International

(cc) BY-NC 